\title{
ULTRAVIOLET OBSERVATIONS OF TRITON IN 1999 WITH THE SPACE TELESCOPE IMAGING SPECTROGRAPH: 2150-3180 ̊ SPECTROSCOPY AND DISK-INTEGRATED PHOTOMETRY
}

\author{
Leslie A. Young and S. Alan Stern \\ Department of Space Studies, Southwest Research Institute, Suite 426, 1050 Walnut Street, Boulder, CO 80302 \\ Received 2000 December 7; accepted 2001 March 13
}

\begin{abstract}
We observed Triton using the Space Telescope Imaging Spectrograph (STIS) on the Hubble Space Telescope (HST) on 1999 August 28, September 11, and September 13 UT. On all three occasions, we obtained images at both 271 and $374 \mathrm{~nm}$, and spectra from 215 to $318 \mathrm{~nm}$. We find that Triton's geometric albedo is high through the near- and mid-UV, decreasing from $0.64 \pm 0.02$ at $374 \mathrm{~nm}$ to $0.53 \pm 0.05$ at $223 \mathrm{~nm}$. We compare these data with a previous UV data set obtained by the HST Faint Object Spectrograph (FOS), taken primarily during 1993 September 1-4. Between 250 and $320 \mathrm{~nm}$, Triton was roughly $20 \%$ brighter in the STIS (1999) data set than in the FOS (1993) data set, but between 220 and $250 \mathrm{~nm}$ the albedos are essentially unchanged. As expected from the HST FOS and other data sets, we find that Triton had higher spatial contrast at shorter wavelengths in 1999, but it exhibited a light-curve amplitude that is as much as 33\% lower than derived from Voyager 2 photometry taken in 1989. We discuss some possible causes for these observed changes.
\end{abstract}

Key words: planets and satellites: individual (Triton) — ultraviolet: solar system

\section{INTRODUCTION}

Neptune's largest satellite, Triton, has a cryogenic $\mathrm{N}_{2} / \mathrm{CH}_{4}$ atmosphere (Broadfoot et al. 1989; Tyler et al. 1989; Gurrola 1995) and a morphologically complex surface (see Smith et al. 1989; Broadfoot et al. 1989; McEwen 1990). Triton's surface, with a temperature near $38 \mathrm{~K}$ (Tyler et al. 1989; Conrath et al. 1989; Tryka et al. 1993; Elliot et al. 2000a), contains the volatile frosts $\mathrm{N}_{2}$, $\mathrm{CO}$, and $\mathrm{CH}_{4}$, as well as less volatile $\mathrm{CO}_{2}, \mathrm{H}_{2} \mathrm{O}$, coloring agents, and absorbers (see, e.g., Thompson \& Sagan 1990; Brown \& Cruikshank 1997; Quirico et al. 1999; Cruikshank et al. 2000). Triton's volatiles are in a continuous state of exchange between the surface and atmosphere, and the transport of volatiles is a probable cause for most of the observed morphological complexity of the surface. Voyager 2 discovered both detached hazes and active vents generating plumes kilometers into Triton's atmosphere (Smith et al. 1989; Broadfoot et al. 1989), indicating an active meteorology on Triton. Adding to the scientific interest in Triton, surface imagery obtained by Voyager 2 in 1989 showed a distinct lack of craters. As a result, Triton's surface is widely thought to be geologically younger and more active than that of almost any other planetary satellite (see, e.g., Smith et al. 1989; Strom, Croft, \& Boyce 1990; Stern \& McKinnon 2000).

Volatile transport on Triton is driven by Triton's orbit and Neptune's obliquity, which together cause extreme seasonal variations in Triton's subsolar latitude (see, e.g., Harris 1984). Because the vapor pressure of the frosts depends exponentially on temperature, changes in insolation lead to large seasonal effects, including wide-scale migration of the volatile frosts and dramatic changes in atmospheric pressure (e.g., Trafton 1984; Spencer 1990). As volatile frosts migrate from sunlit to shaded areas on Triton, they likely expose underlying layers and modify the optical properties of the surface (e.g., Trafton 1984; Spencer $\&$ Moore 1992), affecting Triton's albedo, color, and spectral signature. Because of Triton's high Bond albedo $(A=p q=0.83$; Brown et al. 1995), the surface energy balance is extremely sensitive to these photometric changes.
The observable effects of these predicted changes are expected to be detected as Triton approaches a seasonally driven "major summer" around the start of the 21st century. And indeed, evidence is now mounting that Triton is undergoing a significant period of global seasonal change. One key indicator is the evidence, based on stellar occultations, that Triton's atmospheric pressure has increased since 1989 from 14 to $19 \mu$ bar (Elliot et al. 1998, 2000a, 2000b). Another key indicator is the long-term blueing in Triton's $U-B$ and $B-V$ colors (Buratti et al. 1994).

In addition to these secular changes, transient (e.g., episodic) changes in Triton's color and albedo have also been reported (Buratti et al. 1999; Hicks \& Buratti 2001). These events are marked by temporary but dramatic reddenings of Triton's visible spectral slope, during which the albedo at $3500 \AA$ drops by a factor of $\sim 2$ relative to the albedo at $5500 \AA$. However, because of the sparsity of existing observations, it is unknown how quickly or frequently these changes occur.

Based on the increasing pace and diversity of changes being observed on Triton in the mid-to-late 1990s, and the fact that the UV is usually more sensitive to contrast changes on icy surfaces than are other spectral regions (see, e.g., Stern, Buie, \& Trafton 1997), we conducted a first look for changes in the UV photometric appearance of Triton using the Space Telescope Imaging Spectrograph (STIS) on the Hubble Space Telescope (HST) during HST Cycle 8. This effort consisted of a program of three mid-UV spectroscopic and three concurrent mid-UV imaging observation sets in 1999 August-September, which we present here. In what follows, we describe these observations, present reductions of the data, and then compare the results we obtained with contemporaneous ground-based work obtained by others in 1999 (Hicks \& Buratti 2001), and with previous observations, including other mid-UV HST observations obtained in 1992 and 1993 (Stern, Trafton, \& Flynn 1995) and Voyager 2 UV photometry from 1989 (Lane et al. 1989; Nelson et al. 1990; Hillier et al. 1991). 


\section{HST OBSERVATIONS (1999)}

We observed Triton using the STIS instrument on HST as part of program 8218 ("Exploring Triton in the Act of Global Change") in both imaging and spectroscopic modes. Triton was observed at three approximately equally spaced central meridian longitudes during three visits between 1999 August 28 and September 13. The observing geometry (Table 1) was derived from the JPL DE405 ephemeris (Standish et al. 1995), which used the post-Voyager Triton ephemeris (Jacobson 1990).

Each visit used an identical observing sequence, consisting of one mid-UV spectrum $(1570-3180 \AA)$, four CCD images taken through the F28X50OII filter, centered at $3737 \AA$, and four MAMA images taken through the F25CN270, filter, centered at $2711 \AA$ A (Table 2), for a total of three spectra and 24 images for the entire program. The wavelength range of the spectra was chosen to allow a comparison with earlier mid-UV spectra taken with the Faint Object Spectrograph (FOS) on HST in 1992 and 1993 (Stern et al. 1995). The wavelength for the MAMA images was chosen to allow comparison with earlier spatially resolved images taken with the Faint Object Camera (FOC) on HST in 1995 (Flynn et al. 2001). In particular, the F25CN270 filter was chosen to accentuate albedo changes, balancing the greater spatial contrast on Triton at shorter wavelengths with the higher solar flux above $2600 \AA$ A. Similarly, the F28X50OII filter was chosen to allow comparison with ground-based observations. The STIS images were taken in a dithered pattern to improve spatial resolution; maps of Triton derived from the STIS images will be discussed in a later paper.

Inspection of each of the 24 images showed that Triton was well centered in the detector, with no apparent anomalies. However, to save time during our one-orbit sequences, we did not perform an acquisition of Triton before taking spectra; this resulted in a loss of Triton signal off the edge of the slit. As discussed in detail in $\S 3.3$, we scaled the spectra to agree with albedos derived from the F25CN270 + MAMA images.

\section{ANALYSIS}

\subsection{Overview}

A goal of the reduction for the HST images and spectra is to convert observed count rates or fluxes to disk-integrated geometric albedos, $p(\lambda)$, using the relation

$$
p(\lambda)=\frac{D^{2} \Delta^{2}}{D_{\mathrm{AU}}^{2} R^{2}} \frac{\int F_{\text {Triton }}\left(\lambda^{\prime}\right) \psi\left(\lambda^{\prime}\right) d \lambda^{\prime}}{\int F_{\odot}\left(\lambda^{\prime}\right) \psi\left(\lambda^{\prime}\right) d \lambda^{\prime}} f_{\alpha},
$$

where $D$ and $\Delta$ are the heliocentric and geocentric distances of Triton (Table 1), $R$ is the radius of the target $(1350 \mathrm{~km}$; Smith et al. 1989), $D_{\mathrm{AU}}$ is the mean heliocentric distance of Earth (1 AU), $F_{\text {Triton }}$ is the flux from Triton, $F_{\odot}$ is the flux from the Sun at $1 \mathrm{AU}, \psi$ is the sensitivity of the telescope and instrument (including detector response and filter transmission), and $f_{\alpha}$ is the correction for nonzero solar phase angle. For images, the integrals are performed over the filter bandpasses, as described below. For spectra, the integrals are performed over each wavelength bin. To estimate $f_{\alpha}$ in the mid- and near-UV, we use the linear phase coefficient of $\beta=0.011 \mathrm{mag} \mathrm{deg}^{-1}$ derived at $2500 \AA$ from Voyager images (Lane et al. 1989).

In the mid-UV, the solar flux changes on a daily basis because of the Sun's rotation. For the UV flux of the Sun, $F_{\odot}$, we used the Upper Atmosphere Research Satellite (UARS) Solar-Stellar Irradiance Comparison Experiment (SOLSTICE) solar irradiance spectra for 1999 August 31, September 14, and September 17 for visits 1, 2, and 3, respectively (Woods et al. 1996; T. Woods 2000, private communication). These dates were chosen so that SOLSTICE observed the same face of the Sun that illuminated Triton on our observation dates. The SOLSTICE project initially provided us with preliminary spectra (i.e., version 14) at the instrument resolution of $2.5 \AA$, tabulated on a subsampled grid of $0.5 \AA$, which was used for both the imaging and spectral reductions described below. Later calibration of the solar spectrum (version 15) adjusted solar fluxes by less than $2 \%$ over our spectral range of interest

TABLE 1

Triton ObSERVING GeOMETRy

\begin{tabular}{ccccccc}
\hline \hline Vate & $\begin{array}{c}\text { Central Meridian } \\
\text { Longitude } \\
(\mathrm{deg})\end{array}$ & $\begin{array}{c}\text { Subsolar } \\
\text { Latitude } \\
(\mathrm{deg})\end{array}$ & $\begin{array}{c}\text { Heliocentric } \\
\text { Distance, } D \\
(\text { AU) }\end{array}$ & $\begin{array}{c}\text { Geocentric } \\
\text { Distance, } \Delta \\
(\text { AU) }\end{array}$ & $\begin{array}{c}\text { Solar Phase } \\
\text { Angle, } \alpha \\
(\mathrm{deg})\end{array}$ \\
\hline $1 \ldots \ldots$ & 1999 Aug 28.5 & 8 & -50 & 30.126 & 29.271 & 1.03 \\
$2 \ldots \ldots$ & 1999 Sep 11.2 & 249 & -50 & 30.124 & 29.418 & 1.38 \\
$3 \ldots \ldots$ & 1999 Sep 13.1 & 130 & -50 & 30.124 & 29.442 & 1.42 \\
\hline
\end{tabular}

TABLE 2

Triton ObSERving Summary: EXPOSURE DetaILS

\begin{tabular}{|c|c|c|c|c|c|}
\hline Data Product & Grating/Filter & Detector & 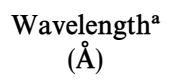 & $\begin{array}{c}\mathrm{FWHM}^{\mathrm{b}} \\
(\AA)\end{array}$ & $\begin{array}{l}\text { Exposure } \\
\text { Time }\end{array}$ \\
\hline One spectrum ............ & G230L & MAMA & $1570-3180$ & $\ldots$ & $1153 \mathrm{~s}$ per spectrum \\
\hline Four dithered images...... & F28X50OII & CCD & 3737.3 & 57 & $50 \mathrm{~s}$ per image \\
\hline Four dithered images...... & $\mathrm{F} 25 \mathrm{CN} 270$ & MAMA & 2710.6 & 160 & $150 \mathrm{~s}_{\text {per image }} \mathrm{c}$ \\
\hline
\end{tabular}

a Wavelengths listed for the images are the pivot wavelength for the corresponding filters, as defined in the HST STIS handbook.

${ }^{\mathrm{b}}$ Full width half-maximum of the filter, as defined in the HST STIS handbook.

${ }^{c}$ The first MAMA image of each visit had an exposure time of $151 \mathrm{~s}$ per image, and the remaining three had exposure times of $150 \mathrm{~s}$ per image. 
TABLE 3

Triton Geometric Albedo: Imaging Results

\begin{tabular}{cccccc}
\hline \hline & Wavelength & & & \\
Filter/Detector & $(\AA)$ & Visit 1 & Visit 2 & Visit 3 & Mean \\
\hline F28X50OII/MAMA ...... & $2631-2791$ & $0.619 \pm 0.004$ & $0.604 \pm 0.003$ & $0.590 \pm 0.005$ & $0.604 \pm 0.028$ \\
F25CN270/CCD ......... & $3709-3766$ & $0.640 \pm 0.005$ & $0.647 \pm 0.005$ & $0.625 \pm 0.008$ & $0.637 \pm 0.020$ \\
\hline
\end{tabular}

NotE.-Errors quoted are $1 \sigma$. Errors for visits 1, 2, and 3 include all systematic errors that affect the relative albedos from visit to visit. Errors for the mean include all known systematic errors that affect comparisons with other data sets (see $\S 3.2)$.

(2200-3800 ̊). Because the version 15 spectra available to us were tabulated on a coarser, $10 \AA$, grid, the albedos we present here used the high-resolution version 14 spectra, scaled to agree with the lower resolution version 15 spectra. The absolute accuracy of the version 15 UARS solar flux is $\sim 1 \%$ from 3000 to $4200 \AA$, and $1 \%-3 \%$ from 1800 to 3000 $\AA$, while the relative accuracy over our 2 week span of observations is estimated to be $0.2 \%$ (T. Woods 2000 , private communication).

The reduction for images and spectra are described individually below, and the resulting albedos are listed in Tables 3 and 4.

\subsection{Images}

For all 24 images, we used the flat-fielded, calibrated data products (the " $\mathrm{flt}$ " files) produced by version 2.0 of the CALSTIS pipeline (Hodge et al. 1998) and performed standard aperture photometry. For the F25CN270 + MAMA images, we used an aperture radius of 0 ".24 (10 pixels) and a background annulus that ranged from 0.36 to 1 ".8 (15 to 75 pixels). For the F28X50OII + CCD images, we used an aperture radius of 0.25 (5 pixels) and a background annulus that ranged from 0 ".5 to 2 ".5 (10 to 50 pixels). For point sources, the fractions of encircled energies for these apertures were 0.85 and 0.92 for the F25CN270 + MAMA and F28X50OII + CCD images, respectively (relative to a $1{ }^{\prime \prime} .0$ aperture; Sahu 1999). Because convolution of the pointspread function (PSF) by a flat disk with Triton's diameter left the encircled energy essentially unchanged, we adopted the point-source encircled energies.

A portion of the observed count rate for the F25CN270 + MAMA images came from a red leak in the F25CN270 filter. This had to be subtracted before further processing. To estimate the count rate due to the flux at wavelengths longer than $3250 \AA$, we used the IRAF/

TABLE 4

Triton Geometric Albedo: Spectral Results

\begin{tabular}{cccc}
\hline \hline $\begin{array}{c}\text { Wavelength } \\
(\AA)\end{array}$ & $\begin{array}{c}\text { Average of } \\
\text { Visits 2 and 3 }\end{array}$ & $\begin{array}{c}\text { Relative } \\
\text { Error }\end{array}$ & $\begin{array}{c}\text { Absolute } \\
\text { Error }\end{array}$ \\
\hline $2150-2310 \ldots \ldots$ & 0.528 & 0.024 & 0.049 \\
$2310-2470 \ldots \ldots$ & 0.524 & 0.019 & 0.049 \\
$2470-2630 \ldots \ldots$ & 0.584 & 0.013 & 0.051 \\
$2630-2790 \ldots \ldots$ & 0.583 & 0.006 & 0.028 \\
$2790-2950 \ldots \ldots$ & 0.631 & 0.006 & 0.029 \\
$2950-3110 \ldots \ldots$ & 0.668 & 0.006 & 0.039 \\
\hline
\end{tabular}

NotE.-Errors quoted are $1 \sigma$. Relative errors include random errors only and should be used for Triton's spectral shape. Absolute errors additionally include all known systematic errors and should be used for comparisons with other data sets (see $\S 3.3)$.
STSDAS SYNPHOT routine. This versatile routine can calculate the predicted count rate through a specified filter given a user-supplied spectrum. In this case, we generated a synthetic spectrum for Triton from 3250 to $7000 \AA$, using visible-wavelength observations of the Sun (Kurucz et al. 1984), the observing geometry of Table 1 , and the nominal $V$ phase coefficient of $0.027 \mathrm{mag} \mathrm{deg}^{-1}$ (Goguen, Hammel, \& Brown 1989). We used an albedo at the wavelengths sensitive to the red leak of 0.7 , based on the long-term measurements of the albedo at $V(5500 \AA)$ by Buratti et al. (1994) and the albedos we measured at 3709-3766 $\AA$ (see Table 3). This red leak contributed $\sim 12 \%$ of the total observed counts in the MAMA images. The uncertainty in the amount of the red-leak contribution is directly related to the uncertainty in the average albedo at the wavelength region of the red leak. As we discuss at the end of $\S 3.3$, Triton's visible albedo was probably stable at a few-percent level over the span of our observations. The uncertainty of the relative albedos of the MAMA images due to the red leak is therefore a few percent of the $12 \%$ red-leak contribution, or less than $0.5 \%$. This error should be used for comparisons within the STIS data set. We can conservatively estimate that we know Triton's absolute visible albedo at the time of the STIS observations to $\pm 10 \%$. The uncertainty of the absolute albedos of the MAMA images due to the red leak is therefore $\sim 10 \%$ of the $12 \%$ red-leak contribution, or less than $1.5 \%$. This error should be used for comparisons of the STIS data set with other data sets.

To convert count rates to geometric albedos, we note that the observed count rate is simply the integral in the numerator in equation (1). For the integral in the denominator, we again used the SYNPHOT routine to calculate the predicted count rate for the UARS solar spectra. ${ }^{1}$ In this calculation we restricted the F25CN270 + MAMA bandpass to wavelengths shortward of $2250 \AA$ because we had already subtracted the red-leak contribution for the F25CN270 + MAMA images. The accuracy of the SYNPHOT calculation is equivalent to the photometric accuracy of STIS: $2.5 \%$ for absolute photometry, and $0.25 \%$ for relative comparisons over our span of observations. The calibrations that support the SYNPHOT cal-

\footnotetext{
${ }^{1}$ An alternative approach to deriving albedos uses the calibration keyword PHOTFLAM to convert the Triton count rate to flux, which is then divided by the solar flux, averaged over a filter bandpass. However, this approach has two drawbacks. First, calculating the average solar flux is complicated by the need to include system throughput and filter response. Second, and more importantly, the calibrations used to generate the PHOTFLAM keyword are based on observations of white dwarfs (C. Proffitt 2000, private communication), which have a different spectral shape over wide filters than does either Triton or the Sun. Therefore, we prefer to ratio count rates rather than fluxes.
} 
culations are normalized to a synthetic aperture of 1.0 (C. Proffitt 2000, private communication), so it is appropriate to ratio the SYNPHOT results to the observed count rates that are adjusted for the encircled energy fraction. The resulting albedos and their $1 \sigma$ errors are listed in Table 3; these errors include random errors, calculated from the scatter of the four images per filter at each visit. As described above, the calibration of the UARS solar flux and the STIS photometric stability contribute additional sources of uncertainty. For the individual visits, Table 3 includes an additional $0.3 \%$ error for the relative error, which should be used for comparisons between visits within the STIS data set. The error in the mean in Table 3 includes an additional $3.9 \%$ uncertainty for the F25CN270 + MAMA images, and a $2.7 \%$ uncertainty for the F28X50OII + CCD images. This larger error should be used for comparisons with other data sets.

The albedos at the three rotational phases differ by several $\sigma$ (see Fig. 1). As derived previously from 1989 Voyager photometry (Hillier et al. 1991) and 1993 HST FOS spectroscopy (Stern et al. 1995), ${ }^{2}$ Triton's UV rotational light-curve amplitude in 1999 is larger through the F25CN270 filter (2631-2791 A) than through the F28X50OII filter (3709-3766 ̊). While the 1993 rotational light-curve amplitude was consistent with the Voyagerderived model (Hillier et al. 1991; Stern et al. 1995), the 1999 STIS data indicate that Triton's mid-UV rotational light curve is lower than the Voyager-derived model, perhaps by as much as $33 \% \pm 13 \%$ at $2631-2791 \AA$ and by $33 \% \pm 20 \%$ at $3709-3766 \AA$. However, given measurements at three longitudes, we can only establish a lower limit to Triton's light-curve amplitude. To crudely assess the significance of the smaller observed amplitude, we modeled how the sparsity of the rotational sampling affects the light-curve amplitude. If we assume that a sinusoidal light curve is

${ }^{2}$ Because eight of the 10 FOS spectra were taken in 1993 September, we assume that the grand-sum average FOS spectrum measures 1993 albedos.

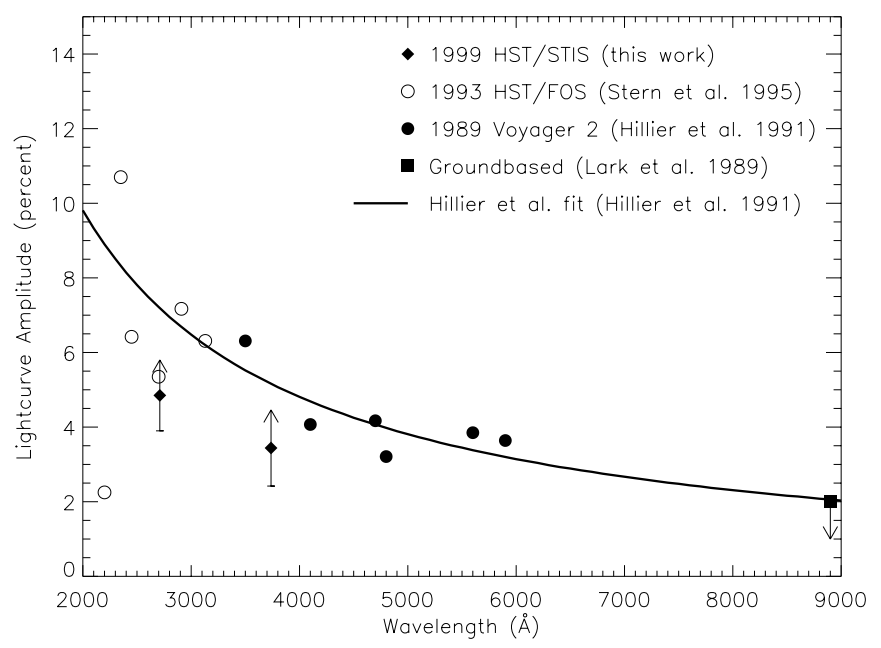

FIG. 1.-Light-curve amplitude vs. wavelength (after Stern, Trafton, \& Flynn 1995). The diamonds represent the light-curve amplitude from STIS, based on measurements at three evenly spaced longitudes. The length of the lower error bar indicates the $1 \sigma$ error of the measurements. The upper portion of the error bar is plotted as an arrow, to indicate that a threepoint light curve generates only a lower limit to the actual light-curve amplitude. sampled at three equally spaced phases, but with a random initial phase, we still find that the amplitude is smaller inn 1999 than it was in 1989 , by $20 \%+14 \%$ at $2631-2791 \AA$, and $21 \% \pm 20 \%$ at $3709-3766 \AA$. Of course, because Triton's light curve may not be sinusoidal, this analysis can only formally suggest that Triton's light curve (especially at 2631-2791 A) is muted in 1999 relative to 1989 or 1993.

Hillier et al. (1991) investigated the time dependence of Triton's light-curve amplitude, for the assumption that the only changes in amplitude were due solely to changing geometry. Because the subobserver latitudes seen from HST in $1993\left(-48^{\circ}\right)$ and $1999\left(-50^{\circ}\right)$ were similar to that seen by Voyager 2 in 1989 for most of the images used by Hillier et al. $\left(-49^{\circ}\right.$ to $\left.-55^{\circ}\right)$, the observed changes in lightcurve amplitude from 1989 and 1993 to 1999 mainly reflect changes in Triton's surface appearance in the UV since 1989 and 1993.

\subsection{Spectra}

One spectrum per visit was taken with the STIS G230L grating and $52^{\prime \prime} \times 0$ ".5 slit, giving a nominal spectral range of 1570-3180 $\AA$ and a nominal dispersion of $1.58 \AA$ pixel $^{-1}$. We used the calibrated data products produced by version 2.0 of the standard CALSTIS pipeline, namely, files of wavelength-calibrated fluxes and errors in ergs $\mathrm{cm}^{-2} \mathrm{~s}^{-1}$ $\AA^{-1}$ (the "x1d" files). These fluxes were low relative to the fluxes derived from the F25CN270 + MAMA images, having only $0.05,0.24$, and 0.30 of the expected flux through the F25CN270 bandpass for visits 1, 2, and 3, respectively. We determined that this is due to a miscentering of the target by $0.3-0.4$, a diagnosis that was confirmed by an observed shift in Triton's wavelength scale relative to the solar spectrum. With the exception of the lower counts and wavelength shifts, the spectra had no obvious anomalies (see Fig. 2). In addition, we confirmed that the CALSTIS pipeline correctly found and extracted the Triton spectrum. Visits 2 and 3, taken within 2 days of each other using the

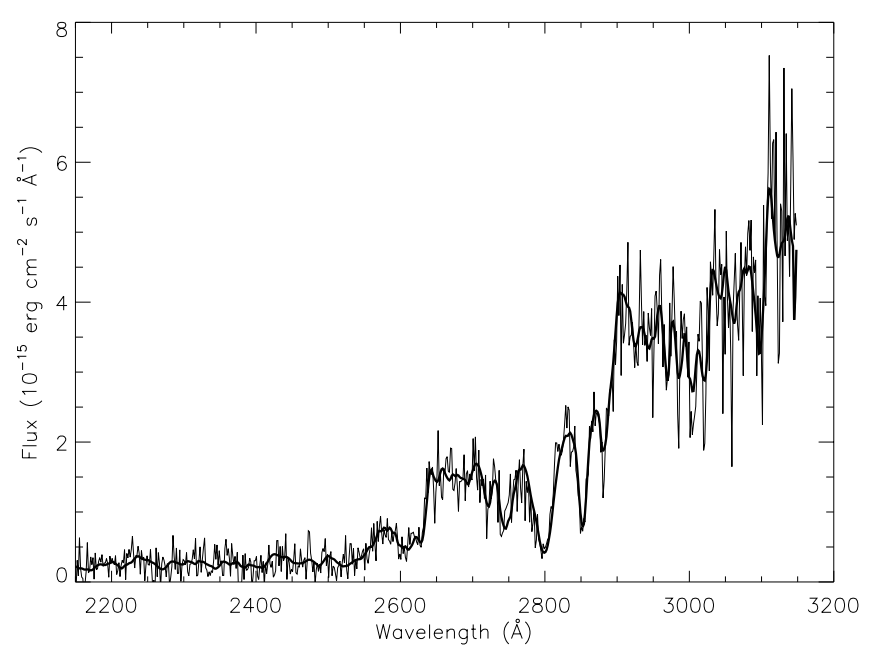

FIG. 2.-Triton and scaled solar fluxes vs. wavelength. Thin line, Triton fluxes from visit 2 , normalized to agree with F25CN270 + MAMA imaging data, plotted at full spectral resolution (1.58 ̊ per point); thick line, the solar flux from $U A R S$, convolved to match the resolution of the STIS spectra and scaled for the derived albedo model for visit 2 . The plotted Triton spectrum is not yet divided by the solar spectrum; all the absorption lines seen in the Triton spectrum are due to reflected sunlight. As expected from the good match between the Triton and scaled solar spectra, division by the solar spectrum removes all solar absorption features cleanly, even at full spectral resolution. 
same HST Guide Star Catalog star for pointing, had similar flux drops and wavelength shifts. Visit 1, taken 2 weeks prior to visit 2 , was slightly farther out of the slit and had a flux drop 5 times worse than that of visits 2 and 3 . Since visit 1 was of much worse quality than the other two, we only report on the spectra from visits 2 and 3 here.

Because the albedos derived from the F25CN270 + MAMA images were derived independently from the spectra, they were unaffected by Triton's miscentering. We therefore normalized the spectra to agree with the F25CN270 + MAMA images. For consistency with the imaging reduction, we determined the normalization by using SYNPHOT with the observed Triton spectra as the input spectrum. The resulting calculated count rates were compared with the observed, adjusted count rates for the F25CN270 + MAMA images. This determined the slit throughput at one particular wavelength. We then calculated the slit throughput as a function of offset distance by integrating a line-spread function (LSF) over an off-center 0.5 region.

We used observations of spectra that were taken as part of an $H S T$ calibration program (program 7721) specifically undertaken to measure the LSF in the cross-dispersion direction. This procedure allowed us to calculate both the offset and the wavelength dependence of the out-of-center slit throughput. In addition, this allowed us to calculate the wavelength shift for an off-center source, confirming miscentering as the cause of the lower flux.

The cross-dispersion direction measured by the LSFs observed in program 7721 was orthogonal to our direction of interest (since Triton was miscentered in the dispersion direction in our STIS observations). To the extent that the STIS PSFs were radially symmetric, the wavelength dependence of the dispersion and cross-dispersion LSFs should be similar. However, the cross-dispersion LSFs were not themselves completely symmetric, and we derived two estimates of the wavelength dependence of the slit throughput, depending on the direction of offset. We used this difference to estimate the error in our derived wavelength dependence of the slit throughput.

The effect of the uncertainty in the slit throughput can be summarized by the following statements: (1) Triton's albedos through the F25CN270 and F28X50OII filters were determined from the STIS imaging data and were therefore independent of the spectral reduction. (2) From 2710 to $2960 \AA$, the slit throughput was nearly constant with wavelength, and independent of the direction of offset. In this wavelength region, the uncertainty in the wavelength dependence of the slit throughput led to an error in the albedo of only $1 \%$, or an error in the slope of the albedo of less than $4 \%$ per $1000 \AA$. (3) From 2200 to $2550 \AA$, the shape of the slit throughput was nearly independent of the direction of offset. Within this wavelength region, the resulting error on the slope of the albedo was less than $3 \%$ per 1000 $\AA$. (4) The relative albedo of the two wavelength regions, 2200-2500 and 2710-2960 $\AA$, was uncertain at the 7\% level.

Once the fluxes were corrected for the out-of-center slit throughput, they were converted to albedos using equation (1). We integrated solar and scaled Triton fluxes over wavelength bins $160 \AA$ wide, where the width of the bin was chosen to match the full width at half-maximum (FWHM) of the F25CN270 filter. For the analysis of the spectra, we assumed that the sensitivity, $\psi$, is approximately constant over the $160 \AA$ wavelength bins (i.e., $\psi$ is a rectangular function). No statistically significant difference in the spectral shape during visits 2 and 3 was detected. We present the mean of visits 2 and 3, and the error in the mean, in Table 4. The relative errors listed are random errors only, propagated from the error vectors provided by the CALSTIS pipeline reduction. The absolute errors include the uncertainties discussed in $\S 3.2$, as well as the wavelength dependence of the slit throughput. No statistically significant spectral features were detected, either at the $160 \AA$ binning of Table 4 or at finer resolution.

In Figure 3, the HST STIS spectra we obtained in 1999 are plotted, along with mid-UV $H S T$ observations obtained in 1992 and 1993 (Stern et al. 1995), the Voyager 2 UV photometry (Nelson et al. 1990), and ground-based work obtained by others in 1999 (Hicks \& Buratti 2001). The STIS spectrum shows a distinct red slope. Because of the issue of the wavelength dependence of the slit throughput, we measured the slope in two regions. In the region from 2710 to $2950 \AA$ (where the slit throughput correction is nearly independent of wavelength), the albedo changes by $42.6 \% \pm 8.9 \%$ per $1000 \AA$. Over the range of $2230-3030 \AA$, the albedo changes by $38.4 \% \pm 7.1 \%$ per $1000 \AA$. As the slopes of visits 2 and 3 differ by only $0.5 \sigma$, Triton shows no detectable evidence for a longitudinal dependence in its mid-UV color.

A striking feature of Figure 3 is the difference between the mid-UV spectroscopy obtained by HST/STIS in 1999 August-September and that obtained by HST/FOS in 1992 and 1993. When quantifying the amount by which Triton has brightened, we find that the errors in the ratio of 1999 albedos to 1993 albedos are dominated by the uncertainty in the overall scaling, namely, uncertainty in the slit throughput, the photometric stability of STIS or FOS, and the random error in the $2710 \AA$ albedo derived here from MAMA images. The sources of uncertainty in the absolute scaling of the STIS MAMA images are discussed in $\S 3.2$,

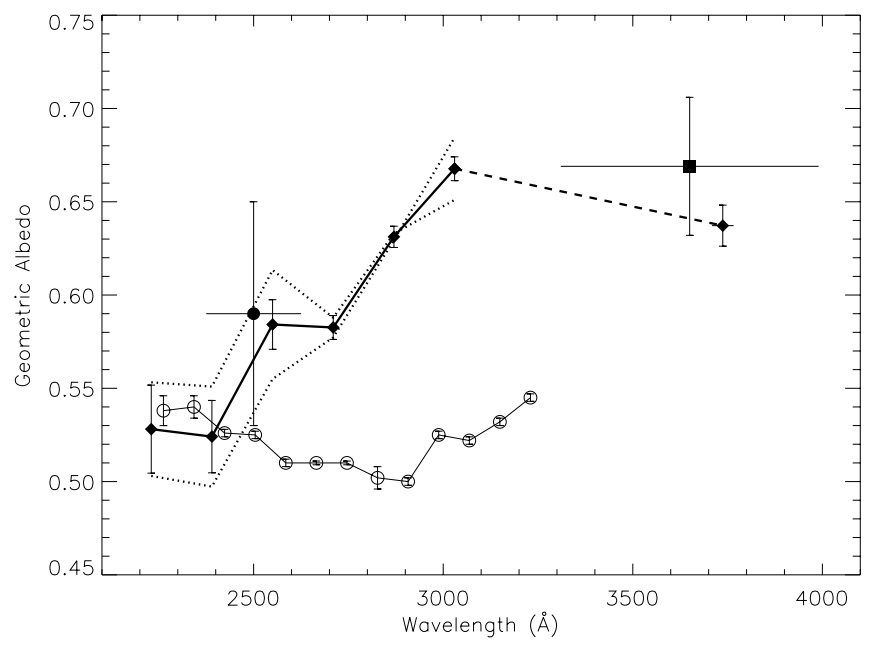

FIG. 3.-UV geometric albedo of Triton, with $1 \sigma$ errors. Albedos from 1999 HST STIS observations are plotted with diamonds and thick lines. The thick dashed line connects the spectral measurements $(2150-3110 \AA$, mean of measurements from September 11 and 13) with the F28X50OII + CCD imaging measurement (3709-3766 A, mean of measurements from August 28, September 11, and September 13). Also plotted are data from 1993, taken with the HST FOS (open circles), data from 1989 taken with the Voyager 2 PPS (filled circle), and ground-based data taken in 1999 (square). The uncertainty in the wavelength dependence of the slit throughput is indicated by the dotted lines. Error bars on STIS and FOS data indicate random errors only (see § 3.3). 
TABLE 5

Triton UV BrightenING, 1993.7 to 1999.7

\begin{tabular}{cc}
\hline \hline $\begin{array}{c}\text { Wavelength Range } \\
(\AA)\end{array}$ & $\begin{array}{c}\text { Ratio of Albedo, } \\
1999 \text { (STIS) to 1993 (FOS) }\end{array}$ \\
\hline $2150-2310 \ldots \ldots \ldots$. & $0.98 \pm 0.13$ \\
$2310-2470 \ldots \ldots \ldots$. & $0.99 \pm 0.13$ \\
$2470-2630 \ldots \ldots \ldots$. & $1.13 \pm 0.15$ \\
$2630-2790 \ldots \ldots \ldots$. & $1.14 \pm 0.13$ \\
$2790-2950 \ldots \ldots \ldots$. & $1.26 \pm 0.14$ \\
$2950-3110 \ldots \ldots \ldots$. & $1.28 \pm 0.15$ \\
\hline
\end{tabular}

NotE.-Errors quoted are $1 \sigma$ and include both random errors and all known systematic errors (see $\S$ 3.3).

and the uncertainties in the wavelength dependence of the slit throughput are discussed above. For the uncertainties in the absolute scaling of the FOS data, we conservatively assumed a relative error of $10 \%$ (Flynn et al. 2001). Triton's mean geometric albedo increased significantly, by $1.26 \pm$ 0.13 at $2870 \AA$ and $1.28 \pm 0.15$ at $3030 \AA$. Between 2200 and $2470 \AA$, however, no change in geometric albedo was detected at the $13 \%$ level (Table 5). As described above, the errors listed in Table 5 include systematic errors in Triton's UV albedo, including photometric stability of STIS and FOS, solar flux calibration, and the error in the redleak correction. These combine to contribute an error of $11 \%$ to the $12 \%-13 \%$ errors in the ratios of Table 5 .

Fortunately, UV spectral shapes and colors are much less susceptible than absolute albedo levels to systematic errors. To look for changes in spectral shape, distinct from changes in overall brightness, we plot the ratio of the $1999 H S T$ STIS spectrum to the 1993 HST FOS spectrum in Figure 4. Doing so reveals that Triton's UV spectrum was redder between 2200 and $3200 \AA$ in 1999 than it was in 1993.

The 2375-2625 $\AA$ albedo obtained by STIS in 1999 August-September $(0.55 \pm 0.04)$ is consistent with Voyager 2 Photopolarimeter Subsystem (PPS) UV photometry at this same wavelength range $(0.59 \pm 0.06$; Nelson et al. 1990). The combination of the HST STIS data and the ground-based $U$ albedo obtained on 1999 August 15 (Hicks \& Buratti 2001) indicates that Triton (1) was not in a

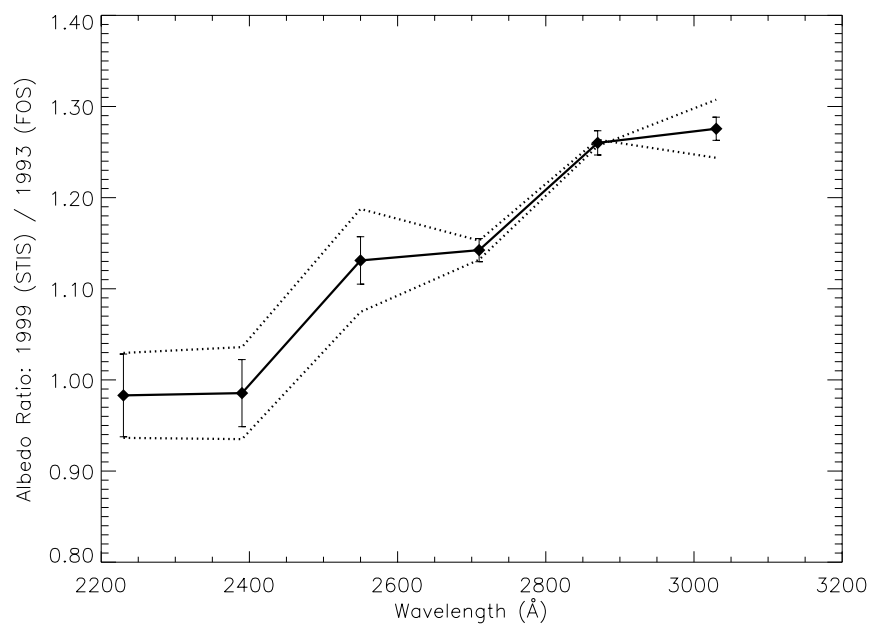

FIG. 4.-Ratio of geometric albedo of Triton in 1999 to that in 1993. Error bars are $1 \sigma$ and indicate random errors only. The uncertainty in the wavelength dependence of the slit throughput is indicated by dotted lines. Uncertainties in the overall scaling of both data sets can adjust the ratios, as a whole, by $11 \%$. reddened state from 1999 August 18 to September 13 and (2) was likely photometrically stable at the few-percent level during this time frame.

\section{DISCUSSION}

As we have shown, Triton was $15 \%-30 \%$ brighter in the mid-UV (2500-3110 $\AA$ ) in 1999 than it was in 1993, and its rotational light-curve amplitude in 1999 appeared smaller than it did in either 1989 or 1993. Furthermore, while it is apparent that Triton was bright and relatively gray from visible to near-UV wavelengths in 1999 , visible-wavelength spectra indicate that Triton was (at times) comparatively much redder and darker in this wavelength region (Buratti, Hicks, \& Newburn 1999).

If Triton's mid- and near-UV albedo is the result of competing brightening and darkening processes, the observed changes indicate a disequilibrium between these processes. Certainly, such a disequilibrium is expected on seasonal (e.g., decadal) timescales, as a result of the transport of volatiles (see, e.g., Trafton 1984), the processing of the $\mathrm{N}_{2} / \mathrm{CO} / \mathrm{CH}_{4}$ frost by radiation (e.g., Salama 1998; Strazzulla 1998), and/or changes in the grain size distribution of frosts (e.g., Eluszkiewicz, Leliwa-Kopystyński, \& Kossacki 1998).

However, changes on much shorter timescales have been observed. For example, the mid-UV changes in spectral shape and albedo that we report here occurred over 6 years. In addition, color changes at near-UV wavelengths are seen on a timescale of years or less from ground-based CCD spectroscopy (Buratti et al. 1999; Hicks \& Buratti 2001). Mid-UV photometry taken with the HST FOC in 1995 (Flynn et al. 2001) and HST/WFPC2 in 1996 (Sromovsky et al. 2001) also apparently indicate that Triton's albedo near $2600 \AA$ can vary dramatically on timescales of 2 years or less. Taken together, these various changes argue for sporadic disequilibrium events, via either the deposition of a widespread coating of a highly reflective material on Triton's surface, or the mechanical underturning or thermally induced submersion of darker, redder coloring agents on Triton's surface. In either case, only a thin $(\sim 100 \mu \mathrm{m})$ layer on the surface is required to significantly alter the photometric properties of the underlying layers.

In the bright-frost deposition case, one can argue against net atmospheric condensation as a source, since stellar occultation data in 1995 and 1997 (e.g., Elliot et al. 2000a) have shown a trend of increasing atmospheric pressure. To have an observable effect between 1993 and 1999, local atmospheric deposition would have to occur preferentially on the areas receiving more sunlight; this is therefore also unlikely. Nevertheless, a plausible mechanism for explaining the observations could be a bright, involatile material (e.g., an aerosol, or perhaps $\mathrm{CO}_{2}$ or $\mathrm{H}_{2} \mathrm{O}$ ice) that is injected into or is chemically generated within the atmosphere, and which then subsequently settles onto the surface. Because Triton's UV albedo below $\sim 2500 \AA$ has remained unchanged since 1993 , this putative material would likely have a reflectance spectrum that declines near $2500 \AA$ (e.g., via an absorption feature, or edge). One source for this material may be Triton's plumes. While the plumes seen during the 1989 Voyager encounter only inject enough bright material to cover $0.4 \%$ of the surface to a depth of $100 \mu \mathrm{m}$ every year (Kirk et al. 1995), the plume activity may well be highly variable in time. Although this sketch scenario can nicely explain the observed changes in Triton's 
UV spectrum between 1993 and 1999, it fails to explain the visible-wavelength data during this period that show Triton switching back and forth from a bluer/grayer, brighter state to a redder, darker state.

In the alternate, dark-material submersion case, material that absorbs in the blue and UV is being deposited on the surface from time to time, which modifies this portion of Triton's spectrum. In such a scenario, the "events" are the reddenings, rather than the brightenings, and the subsequent brightening of Triton occurs as the red material somehow "sinks" (e.g., thermally, because of its lower albedo) or is otherwise buried below the optically active layer (e.g., perhaps mechanically). Potential candidates for this red material include carbon- or sulfur-bearing compounds, tholins, and other aerosols, created in the atmosphere or in plume sources.

Combining these two scenarios, it might be that the dark material was laid down in thin, intermittent layers with bright condensing frosts during previous periods of decreasing atmospheric pressure on Triton. During one deposition season, a thick slab of alternating layers may be produced. In this scenario, the increasing atmospheric pressure now seen is successively exposing dark material as the bright, volatile frost layers between the dark-material layers are sublimated into the atmosphere. In order to explain the observed rapid changes in Triton's UV and blue albedo, the layers in the topmost "Triton layer cake" (or TLC) must themselves be quite thin. For example, the $5 \mu$ bar increase of the surface pressure that appears to have occurred between 1989 and 1997 (Elliot et al. 2000a) would strip off a global average of only $1000 \mu \mathrm{m}$. Although this depth is thin on geologic scales, it is certainly thick enough to obscure the photometric properties of underlying layers.

Clearly, there exist many alternative explanations for the observed changes, because the problem is as yet underconstrained. Observations to better characterize the frequency, transition time, and duration of reddening of brightening events, the albedo and color distribution of Triton before, during, and after such events, and the ongoing atmospheric pressure changes would be extremely helpful for discriminating between these alternatives.

\section{CONCLUSIONS}

In summary, the results obtained above lead to the following conclusions:

1. Comparing the mid-UV (2150-3180 $\AA$ ) spectroscopy obtained by HST/STIS in 1999 August-September with the mid-UV (1900-3278 A) spectroscopy obtained by $H S T / F O S$ in 1993 September (Stern et al. 1995), we find that Triton's mid-UV geometric albedo increased significantly, from $26 \% \pm 14 \%$ at $2790-2950 \AA$ to $28 \% \pm 15 \%$ at 2950-3110 ̊. Between 2200 and $2500 \AA$, no change in broadband geometric albedo was detected at the $13 \%$ level. The dramatic change at longer wavelengths, combined with the lack of observable change at shorter wavelengths, provides important constraints on the nature of the agents creating the change.

2. When the mean 1999 STIS spectrum is divided by the 1993 FOS spectrum, we find that Triton's spectrum in the mid-UV (2150-3180 $\AA$ ) has reddened.

3. Comparing the mid-UV photometry at $2375-2624 \AA$ obtained by STIS in $1999(0.55 \pm 0.04)$ with the photometry at the same wavelength range obtained by the Voyager 2 PPS in $1989(0.59 \pm 0.06$; Nelson et al. 1990), we find no change at the $9 \%$ level.

4. Consistent with the 1993 FOS mid-UV spectroscopy, we find that Triton's UV rotational light-curve amplitude is larger through the F25CN270 filter (2631-2791 ̊) than through the F28X50OII filter (3709-3766 ̊). The amplitudes are lower than predicted by the model of Hillier et al. (1991), by up to $33 \% \pm 13 \%$ at $2631-2791 \AA$ and $33 \% \pm 20 \%$ at $3709-3766 \AA$, although our sparse rotational sampling accounts for some of this lower amplitude.

5. The combination of the HST STIS data and the ground-based $U$ albedo obtained on 1999 August 15 (Hicks \& Buratti 2001) indicates that Triton (a) was not in a reddened state from 1999 August 18 to September 13 and (b) was likely photometrically stable at the few-percent level during this time frame.

The results we have reported here demonstrate that Triton's UV photometric properties have changed in several key respects between 1989, 1993, and 1999. We interpret this as a consequence of seasonal and/or transient changes, most likely due to volatile transport or plume activity. As with visible-wavelength photometry and occultation-based probes of Triton's atmospheric structure, more frequent and rotationally denser measurements are required in the UV in order to determine or constrain the source of Triton's photometric changes.

Marc Buie kindly performed the aperture photometry to calculate background-subtracted counts for the imaging data. Our colleagues Larry Trafton and Joel Parker contributed significantly to the success of this $H S T$ program. Many thanks to Tom Woods for providing the UARS SOLSTICE spectra. Alex Storrs pointed out the utility of SYNPHOT. We especially thank Charles Proffitt; this project could not have been completed without his timely and useful information on line-spread functions and encircled energies. The NSO/Kitt Peak FTS data used here were produced by NOAO. This work was supported, in part, under STScI grants AR-08349.07-97A and GO-08218.0197A. The Hubble Space Telescope is operated for NASA by the Space Telescope Science Institute (STScI), a division of AURA, Inc.

\section{REFERENCES}

Broadfoot, A. L., et al. 1989, Science, 246, 1459

Brown, R. H., \& Cruikshank, D. P. 1997, Ann. Rev. Earth Planet. Sci., 25, 243

Brown, R. H., Cruikshank, D. P., Veverka, J., Helfenstein, P., \& Eluszkiewicz, J. 1995, in Neptune and Triton, ed. D. P. Cruikshank (Tucson: Univ. Arizon. Press), 991

Buratti, B. J., Goguen, J. D., Gibson, J., \& Mosher, J. 1994, Icarus, 110, 303

Buratti, B. J., Hicks, M. D., \& Newburn, R. L., Jr. 1999, Nature, 397, 219

Conrath, B., et al. 1989, Science, 246, 1454

Cruikshank, D. P., et al. 2000, Icarus, 147, 309

Elliot, J. L., et al. 1998, Nature, 393, 765
Elliot, J. L., et al. 2000a, Icarus, 148, 347

Elliot, J. L., Strobel, D. F., Zhu, X., Stansberry, J. A., Wasserman, L. H., \& Franz, O. G. 2000b, Icarus, 143, 425

Eluszkiewicz, J., Leliwa-Kopystyński, J., \& Kossacki, K. J. 1998, in Solar System Ices, ed. B. Schmitt, C. de Bergh, \& M. Festou (Dordrecht: Kluwer), 119

Flynn, B., Stern, S. A., Trafton, L., \& Stansberry, J. 2001, Icarus, 150, 297

Goguen, J. D., Hammel, H. B., \& Brown, R. H. 1989, Icarus, 77, 239

Gurrola, E. M. 1995, Ph.D. thesis, Stanford Univ.

Harris, A. W. 1984, in Uranus and Neptune, ed. J. T. Bergstralh (NASA CP-2330) (Washington: NASA), 357

Hicks, M. D., \& Buratti, B. J. 2001, in preparation 
Hillier, J., Veverka, J., Helfenstein, P., \& McEwen, A. 1991, J. Geophys. Res. Suppl., 96, 19211

Hodge, P. E., et al. 1998, in ASP Conf. Ser. 145, Astronomical Data Analysis Software and Systems VII, ed. R. Albrecht, R. N. Hook, \& H. A. Bushouse (San Francisco: ASP), 316

Jacobson, R. A. 1990, A\&A, 231, 241

Kirk, R. L., Soderblom, L. A., Brown, R. H., Kieffer, S. W., \& Kargel, J. S. 1995, in Neptune and Triton, ed. D. P. Cruikshank (Tucson: Univ. Arizona Press), 949

Kurucz, R. L., Furenlid, I., Brault, J., \& Testerman, L. 1984, Solar Flux Atlas from 296 to $1300 \mathrm{~nm}$ (Sunspot, NM: Natl. Sol. Obs.)

Lane, A. L., et al. 1989, Science, 246, 1450

Lark, N., Hammel, H. B., Cruikshank, D. P., Tholen, D. J., \& Rigler, M. A. 1989, Icarus, 79, 15

Livingston, W., Donnelly, R. F., Grigoryev, V., Demidov, M. L., Lean, J. Steffen, M., White, O. R., \& Wilson, R. L. 1991, in Solar Interior and Atmosphere, ed. A. N. Cox, W. C. Livingston, \& M. S. Matthews (Tucson: Univ. Arizona Press), 1109

McEwen, A. S. 1990, Geophys. Res. Lett., 17, 1765

Nelson, R. M., Buratti, B. J., Wallis, B. D., Smythe, W. D., \& Horn, L. J. 1990, Geophys. Res. Lett., 17, 1761

Quirico, E., Douté, S., Schmitt, B., de Bergh, C., Cruikshank, D. P., Owen, T. C., Geballe, T. R., \& Roush, T. L. 1999, Icarus, 139, 159

Sahu, K., ed. 1999, Space Telescope Imaging Spectrograph Instrument Handbook (version 3.0; Baltimore: STScI), chap. 14
Salama, F. 1998, in Solar System Ices, ed. B. Schmitt, C. de Bergh, \& M. Festou (Dordrecht: Kluwer), 259

Smith, B. A., et al. 1989, Science, 246, 1422

Spencer, J. R. 1990, Geophys. Res. Lett., 17, 1769

Spencer, J. R., \& Moore, J. M. 1992, Icarus, 99, 261

Sromovsky, L. A., Fry, P. M., Baines, K. H., \& Dowling, T. E. 2001, Icarus, 149,435

Standish, E. M., Newhall, X X, Williams, J. G., \& Folkner, W. M. 1995 , JPL Planetary and Lunar Ephemerides, DE403/LE403 (Interoffice Memo. 314.10-127) (Pasadena: JPL)

Stern, S. A., Buie, M. W., \& Trafton, L. M. 1997, AJ, 113, 827

Stern, S. A., \& McKinnon, W. B. 2000, AJ, 119, 945

Stern, S. A., Trafton, L. M., \& Flynn, B. 1995, AJ, 109, 2855

Strazzulla, G. 1998, in Solar System Ices, ed. B. Schmitt, C. de Bergh, \& M. Festou (Dordrecht: Kluwer), 281

Strom, R. G., Croft, S. K., \& Boyce, J. M. 1990, Science, 250, 437

Thompson, W. R., \& Sagan, C. 1990, Science, 250, 415

Trafton, L. 1984, Icarus, 58, 312

Tryka, K. A., Brown, R. H., Anicich, V., Cruikshank, D. P., \& Owen, T. C. 1993, Science, 261, 751

Tyler, G. L., et al. 1989, Science, 246, 1466

Woods, T. N., et al. 1996, J. Geophys. Res., 101, 9541 